\title{
Peripheral blood mononuclear cells preferentially activate 11-oxygenated androgens
}

\section{Lina Schiffer ${ }^{\mathbb{1} 1}$, Alicia Bossey1, Punith Kempegowda ${ }^{\mathbb{1} 1}$, Angela E Taylor1, Ildem Akerman', Dagmar Scheel-Toellner², Karl-Heinz Storbeck ${ }^{1,3}$ and Wiebke Arlt $\mathbb{D} 1,4$}

${ }^{1}$ Institute of Metabolism and Systems Research, ${ }^{2}$ Institute of Inflammation and Ageing, University of Birmingham, Birmingham, UK, ${ }^{3}$ Department of Biochemistry, Stellenbosch University, Stellenbosch, South Africa, and ${ }^{4} \mathrm{National}$ Institute for Health Research (NIHR), Birmingham Biomedical Research Centre, University Hospitals Birmingham

Correspondence should be addressed to W Arlt Email w.arlt@bham.ac.uk

\begin{abstract}
Objective: Androgens are important modulators of immune cell function. The local generation of active androgens from circulating precursors is an important mediator of androgen action in peripheral target cells or tissues. We aimed to characterize the activation of classic and 11-oxygenated androgens in human peripheral blood mononuclear cells (PBMCs). Methods: PBMCs were isolated from healthy male donors and incubated ex vivo with precursors and active androgens of the classic and 11-oxygenated androgen pathways. Steroids were quantified by liquid chromatography-tandem mass spectrometry. The expression of genes encoding steroid-metabolizing enzymes was assessed by quantitative PCR. Results: PBMCs generated eight-fold higher amounts of the active 11-oxygenated androgen 11-ketotestosterone than the classic androgen testosterone from their respective precursors. We identified the enzyme AKR1C3 as the major reductive $17 \beta$-hydroxysteroid dehydrogenase in PBMCs responsible for both conversions and found that within the PBMC compartment natural killer cells are the major site of AKRC13 expression and activity. Steroid 5 $\alpha$-reductase type 1 catalyzed the $5 \alpha$-reduction of classic but not 11-oxygenated androgens in PBMCs. Lag time prior to the separation of cellular components from whole blood increased serum 11-ketotestosterone concentrations in a time-dependent fashion, with significant increases detected from two hours after blood collection.

Conclusions: 11-Oxygenated androgens are the preferred substrates for androgen activation by AKR1C3 in PBMCS, primarily conveyed by natural killer cell AKR1C3 activity, yielding 11-ketotestosterone the major active androgen in PBMCs. Androgen metabolism by PBMCs can affect the results of serum 11-ketotestosterone measurements, if samples are not separated in a timely fashion.
\end{abstract}

\section{Significance statement}

We show that human peripheral blood mononuclear cells (PBMCs) preferentially activate 11-ketotestosterone rather than testosterone when incubated with precursors of both the classic and the adrenal-derived 11-oxygenated androgen biosynthesis pathways. We demonstrate that this activity is catalyzed by the enzyme AKR1C3, which we found to primarily reside in natural killer cells, major contributors to the anti-viral immune defense. This potentially links intracrine 11-oxygenated androgen generation to the previously observed decreased NK cell cytotoxicity and increased infection risk in primary adrenal insufficiency. In addition, we show that PBMCs continue to generate 11-ketotestosterone if the cellular component of whole blood samples is not removed in a timely fashion, which could affect measurements of this active androgen in routine clinical biochemistry. 


\section{Introduction}

A critical step in androgen activation is the conversion of the androgen precursor androstenedione (A4) to the active androgen testosterone (T) $(1,2,3)$, which occurs in the testes catalyzed by $17 \beta$-hydroxysteroid dehydrogenase type 3 (HSD17B3). However, in the majority of peripheral target tissues of androgen action, the intracrine activation of A4 to $\mathrm{T}$ is catalyzed by the enzyme AKR1C3 (aldoketo reductase 1C3, also termed 17 $\beta$-hydroxysteroid dehydrogenase type 5) $(1,2,3)$, which thereby represents a key regulator of local androgen exposure. Peripheral blood mononuclear cells (PBMCs) can activate A4 to T $(4,5)$ catalyzed by AKR1C3 (6). Androgens impact on a multitude of immune cell functions, including proliferation, differentiation, apoptosis, cytokine and immunoglobulin productions $(7,8)$ and also link immune and metabolic regulation (9).

Recent in vitro work has shown that AKR1C3 has an approximately eight-fold higher catalytic efficiency for the generation of the 11-oxygenated androgen 11-ketotestosterone (11KT) from its precursor 11-ketoandrostenedione (11KA4) than for the generation of $\mathrm{T}$ from A4 (10). 11KT activates the androgen receptor with potency and efficacy similar to $T(11,12,13)$ and is the predominant circulating androgen in androgen excess conditions including congenital adrenal hyperplasia, polycystic ovary syndrome and premature adrenarche $(12,14,15) .11 \mathrm{KT}$ is derived from the abundant adrenal precursor $11 \beta$-hydroxyandrostenedione (11OHA4) $(13,16)$, which is converted to 11KA4 in peripheral tissues expressing 11ß-hydroxysteroid dehydrogenase type 2 (HSD11B2) (17, 18) prior to activation to $11 \mathrm{KT}$ by AKR1C3 $(10,16)$.

Of note, a number of studies have shown gradual increases in $\mathrm{T}$ measured in serum, if the blood sample is left unseparated from cellular components for an increased period of time, using either immunoassays (19, 20,21 ) or ultra-high performance liquid chromatographytandem mass spectrometry (LC-MS/MS) for steroid quantification $(22,23)$. One of those studies additionally investigated the effect of time before separation on $11 \mathrm{KT}$ concentrations measured in serum and observed a much more pronounced increase in $11 \mathrm{KT}$ than $\mathrm{T}$ over time (23), which could have a differential impact on the measurement of circulating 11-oxygenated androgen concentrations.

In this study, we explore this further and undertake a detailed characterization of androgen activation via both classic and 11-oxygenated androgen biosynthesis pathways in the human PBMC compartment and subpopulations, utilizing PBMCs isolated from healthy volunteers.

\section{Subjects and methods}

\section{Blood collection}

The collection of blood for PBMC isolation and serum steroid analysis underwent ethical approval by the Science, Technology, Engineering and Mathematics Ethical Review Committee of the University of Birmingham (ERN_17-0494, ERN_17-0494A, and ERN_14-0446). All participants gave informed, written consent prior to study participation. Exclusion criteria for participation were any acute or chronic disease affecting steroid biosynthesis or metabolism and intake of any medication known to interfere with steroid biosynthesis or metabolism. All blood samples were collected between 09:00 $\mathrm{h}$ and 11:00 h; $50 \mathrm{~mL}$ of blood were collected into $\mathrm{K}_{2}$ EDTA Vacutainers ${ }^{\mathrm{TM}}$ (purple top) for the immediate isolation of PBMCs.

To assess the effect of the time period between blood collection and separation of the cellular components by centrifugation on the measured concentrations of serum steroids, blood was collected from healthy volunteers (3 male, 3 female; age range: $28-50$ years; BMI range 20.8 $31.1 \mathrm{~kg} / \mathrm{m}^{2}$ ) into several SST ${ }^{\mathrm{TM}}$ Vacutainers ${ }^{\mathrm{TM}}$ (gold top). The tubes were left unseparated at room temperature in an air-conditioned room and were separated by centrifugation $(2000 \mathrm{~g}, 10 \mathrm{~min})$ after defined time periods $(0,1,2,4,6$ and $24 \mathrm{~h})$. The separated serum was stored at $-80^{\circ} \mathrm{C}$.

\section{Isolation of peripheral blood mononuclear cells}

PBMCs were isolated from whole blood by density gradient centrifugation using Ficoll Paque Plus (GE Healthcare) following the manufacturer's instructions. Isolated PBMCs were washed in RPMI-1640 (SigmaAldrich) supplemented with $100 \mathrm{U} / \mathrm{mL}$ penicillin and 0.1 $\mathrm{mg} / \mathrm{mL}$ streptomycin, counted and assessed for viability by trypan blue exclusion. A minimum of $3 \times 10^{6}$ cells was frozen in $400 \mu \mathrm{L}$ TRI reagent ${ }^{\circledR}$ (Sigma Aldrich) and stored at $-80^{\circ} \mathrm{C}$ for RNA analysis at a later date.

\section{RNA extraction and quantitative PCR}

Samples stored in TRI reagent ${ }^{\circledR}$ were defrosted and the RNA in the aqueous phase of the phenol-chloroform extraction was purified using the RNeasy Mini Kit (Qiagen). RNA concentrations were determined from the 
absorbance of the sample at $260 \mathrm{~nm}$ using a Nanodrop spectrophotometer and reverse transcription (RT) was performed using Applied Biosystems ${ }^{\mathrm{TM}}$ TaqMan $^{\mathrm{TM}}$ RT Reagents following the manufacturer's protocol. Quantitative PCR (qPCR) was performed on an ABI 7900HT sequence detection system (Perkin Elmer, Applied Biosystems) using TaqMan ${ }^{\mathrm{TM}}$ Gene Expression Assays (FAM-labeled) and the SensiFAST TM Probe Hi-ROX kit (Bioline). $\triangle \mathrm{Ct}$ was calculated as $\mathrm{Ct}$ (Target)-Geometric mean (Ct (HPRT1), Ct (GAPDH)). Gene expression in arbitrary units (A.U.) was calculated as $1000^{*} 2^{-\Delta \mathrm{Ct}}$. For targets not reproducibly detected in duplicate reactions, relative gene expression is shown as 0 .

\section{Ex vivo steroid metabolism assays in peripheral blood mononuclear cells}

For steroid metabolism assays, $3 \times 10^{6}$ cells were incubated with $100 \mathrm{nM}$ of the respective steroid in a final volume of $500 \mu \mathrm{L}$ RPMI-1640 supplemented with penicillin and streptomycin. Steroids were added from stock solutions in methanol. The final methanol concentration in the incubations was $0.00304 \%$ (v/v). Technical replicates were prepared, if sufficient cell numbers were available. During the incubation, the samples were constantly gently rotated at $37^{\circ} \mathrm{C}$ for $24 \mathrm{~h}$. For each experiment, incubations of cells with methanol only and cell-free incubations for all steroids were prepared as controls. Additionally, dilutions of each steroid at $100 \mathrm{nM}$ in medium were frozen at $-20^{\circ} \mathrm{C}$ immediately after preparation. At the end of the incubation period samples were centrifuged for $2 \mathrm{~min}$ at 9,400 rpm. The supernatant was stored at $-20^{\circ} \mathrm{C}$ for steroid analysis. The cell pellet was washed in PBS, suspended in $50 \mu \mathrm{L}$ lysis buffer (50 mM Tris pH 8.0, $150 \mathrm{mM} \mathrm{NaCl}, 0.1 \%$ SDS, $0.5 \%$ sodium deoxycholate, $1 \%$ Trition X-100, $0.1 \mathrm{mM}$ DTE, $0.1 \mathrm{mM}$ PMSF, $0.1 \mathrm{mM}$ EDTA) and stored at $-80^{\circ} \mathrm{C}$ for protein quantification.

\section{Steroid analysis by liquid chromatography-tandem mass spectrometry}

The following steroids were quantified by LC-MS/MS in the supernatant of the PBMC incubations after centrifugation and in serum samples as previously described (15): $11 \beta$-hydroxy- $5 \alpha$-androstanedione

(11OH-5 $\alpha$-dione, $5 \alpha$-androstan-11 $\beta$-ol-3,17-dione), 11OHA4 (4-androsten11 $\beta$-ol-3,17-dione), 11 $\beta$-hydroxytestosterone (11OHT, $\quad 4$-androstene-11 $\beta, 17 \beta$-diol-3-one), 11KA4 (4-androsten-3,11,17-trione), $11 \mathrm{KT} \quad$ (4-androsten17 $\beta$-diol-3,11-dione), $5 \alpha$-dihydrotestosterone (DHT,
$5 \alpha$-androstan-17 $\beta$-ol-3-one), $5 \alpha$-androstanediol (Adiol, $5 \alpha$-androstan-3 $\alpha, 17 \beta$-diol), $\quad 5 \alpha$-androstanedione (5 $\alpha$-dione, $5 \alpha$-androstan-3,17-dione), A4 (4-androsten3,17-dione), androsterone (An, 5 $\alpha$-androstan3 $\alpha$-ol-17-one), dehydroepiandrosterone (DHEA, 5-androsten-3 $\beta$-ol-17-one), T (4-androsten-17 $\beta$-ol-3one). In addition, 11-keto-5 $\alpha$-dihydrotestosterone (11KDHT, $5 \alpha$-androstan-17 $\beta$-ol-3,11-dione) and 11-keto$5 \alpha$-androstanedione ( $5 \alpha$-androstan-3,11,17-dione) were analyzed by ultra-high performance supercritical fluid chromatography-tandem mass spectrometry as previously described (24). For ex vivo cell incubations, steroid concentrations or their ratios were normalized to the total protein content of the incubations as determined in the supernatant after cell lysis using the DC Protein Assay (Bio-Rad).

\section{Analysis of published RNAseq data from PBMC subpopulations}

We accessed dice-database.org (25) to investigate AKR1C3 expression determined by RNAseq in FACS-sorted PBMC subpopulations. The database contains expression data from 54 male and 37 female healthy donors (age range 18-61 years). AKR1C3 expression data were downloaded and plotted in GraphPad Prism 8 as $\log _{2}$ transformed transcripts per million (TPM).

\section{Natural killer cell ex vivo steroid metabolism assays}

PBMCs were prepared as described above and NK cells were subsequently enriched using the MACS ${ }^{\circledR}$ human NK cell isolation kit (Miltenyi Biotec) as per the manufacturer's instructions. Cells were checked for viability, counted and steroid conversion assays were set up as described above.

\section{Statistical analysis}

Changes in serum steroid concentrations were analyzed in GraphPad Prism 8 using ANOVA followed by a Dunnett multiple comparison test to compare each timepoint against the sample processed immediately after collection $(0 \mathrm{~h})$. Statistical analysis of differences in steroid concentrations in the supernatants from the PBMC ex vivo incubations was performed by Wilcoxon matched-pairs signed-rank test or Mann-Whitney test as appropriate. Statistical analysis of RNAseq data was performed by oneway ANOVA followed by Tukey's multiple comparisons test or Mann-Whitney test as appropriate. 


\section{Results}

\section{Androgen activation in PBMCs is primarily catalyzed by AKR1C3 and SRD5A1}

Using qPCR, we identified $A K R 1 C 3$ as the major reductive, activating $17 \beta$-hydroxysteroid dehydrogenase isoform in PBMCs, while $H S D 17 B 3$, generally considered a testesspecific isoform, was expressed at very low levels only (Fig. 1A). While we could not detect the expression of steroid $5 \alpha$-reductase type 2 (SRD5A2) and 17 $\beta$-hydroxysteroid dehydrogenase type 2 (HSD17B2) in any of the samples from 14 donors using our qPCR assay, we detected consistently high expression of steroid $5 \alpha$-reductase type 1 (SRD5A1) in samples from all donors $(n=14)$ and detected 17ß-hydroxysteroid dehydrogenase type 4 (HSD17B4) expression in samples from 6 of 14 donors. This indicates that SRD5A1 is responsible for the $5 \alpha$-reduction we observe in our PBMC incubations with androgen substrates while HSD17B4 catalyzes the oxidative, inactivating $17 \beta$-hydroxysteroid dehydrogenase activity we observed (Fig. 1A), consistent with previously published findings (6). 11ß-hydroxysteroid dehydrogenase type 1 (HSD11B1) expression was detectable in samples from 13/14 donors, while HSD11B2 mRNA was not detected. Figure 1B schematically illustrates the enzymes identified by qPCR as present in PBMCs and their roles in androgen activation and inactivation in the classic and 11-oxygenated androgen biosynthesis pathways.

\section{PBMCs preferentially activate 11-oxygenated androgens}

In order to unravel the pathways of androgen metabolism in PBMCs, we isolated PBMCs from male healthy donors and performed ex vivo incubations, using as substrates several androgen precursors and active androgens from both the classic (DHEA, A4 and T) and 11-oxygenated androgen pathways (11OHA4, 11OHT, 11KA4, 11KT). Product formation was quantified by LC-MS/MS.

Initial time-course experiments with PBMCs isolated from three male donors (aged 22, 23 and 28 years) identified $24 \mathrm{~h}$ as a suitable incubation time allowing for robust product quantification within the linear range of product formation over time (data not shown). These initial experiments additionally revealed very low $3 \beta$-hydroxysteroid dehydrogenase activity in PBMCs and thus excluded DHEA as a relevant substrate for metabolism in PBMCs (data not shown).

All further experiments were performed with PBMCs isolated from men aged $18-30$ years $(n=4-5$; age $22-30$ years; BMI $20.2-29.1 \mathrm{~kg} / \mathrm{m}^{2}$ ) and men aged $>50$ years $\left(n=4-7\right.$; age $53-72$ years; BMI $\left.21.2-30.4 \mathrm{~kg} / \mathrm{m}^{2}\right)$. We did not observe any significant differences in product formation by PBMCs between the two different age groups (Fig. 2), except a slightly, but not significantly increased median formation of T from A4 and 11KT from 11KA4. Hence, we present and discuss the combined data for the entire cohort below ( $n=8-12$ per experiment; age range 22-72; BMI range $20.2-30.4 \mathrm{~kg} / \mathrm{m}^{2}$ ).

Incubations with $\mathrm{A} 4$ and $\mathrm{T}$ yielded their respective $5 \alpha$-reduced products, $5 \alpha$-dione and DHT, and revealed efficient interconversion of $\mathrm{A} 4$ and $\mathrm{T}$ by reductive and oxidative 17 $\beta$-hydroxysteroid dehydrogenases (Fig. 3A and B). $5 \alpha$-dione and DHT were further converted to their $3 \alpha$-hydroxy metabolites An and Adiol.

The quantitatively dominant formation observed among all substrates tested was the generation of $11 \mathrm{KT}$ from 11KA4, which was significantly higher than the generation of T from A4 ( $P=0.001 ; n=11$; Fig. 3C). After an incubation period of $24 \mathrm{~h}$, PBMCs generated approximately eight

\section{A}

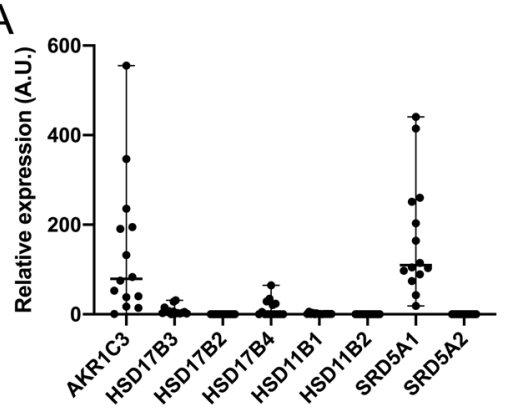

B

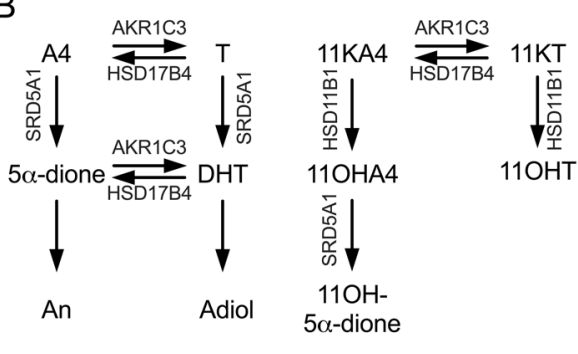

\section{Figure 1}

Expression of genes encoding key androgen-metabolizing enzymes in PBMCs isolated from healthy men ( $A, n=14$; age range 22-72 years; BMI range 20.2-30.4 kg/ $\mathrm{m}^{2}$ ). Gene expression was assessed by quantitative PCR and normalized to HPRT1 and GAPDH expression. Arbitrary units (A.U.) were calculated as $1000 \times 2^{-\Delta C t}$. (B) Schematic representation of the steroidmetabolizing enzymes in PBMCs and their roles in activation and inactivation of androgens in the classic and 11-oxygenated androgen pathways. 
A
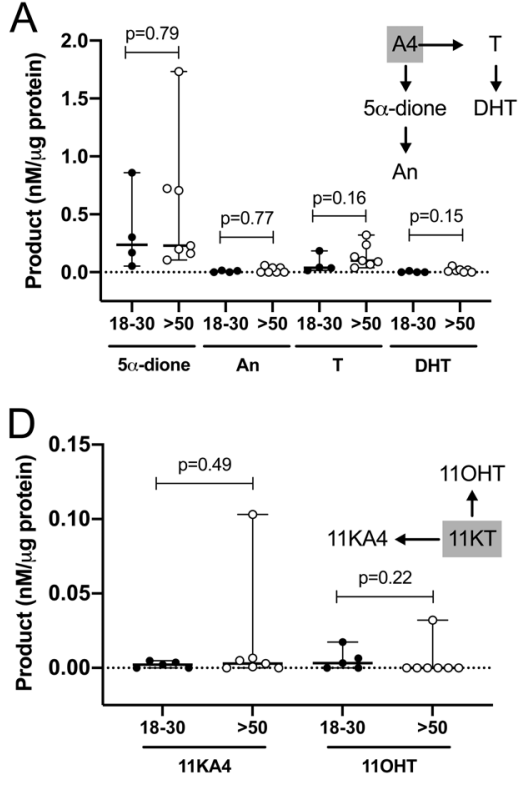

$B$

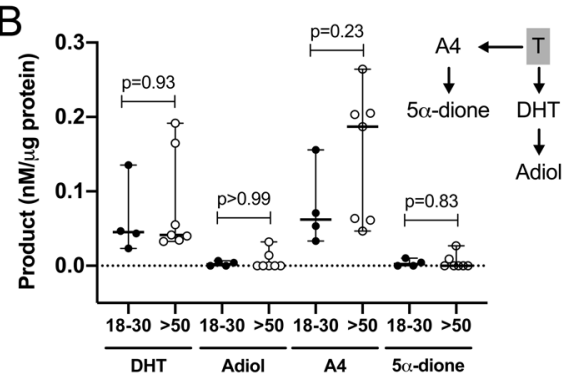

E

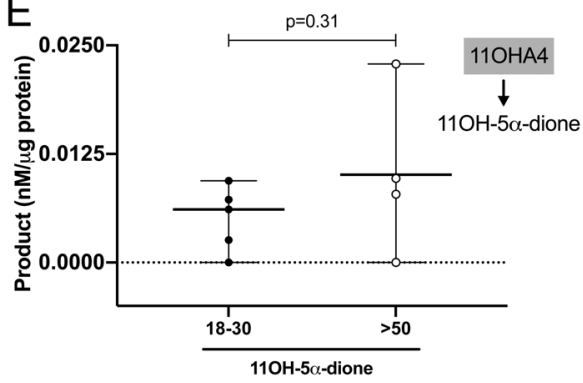

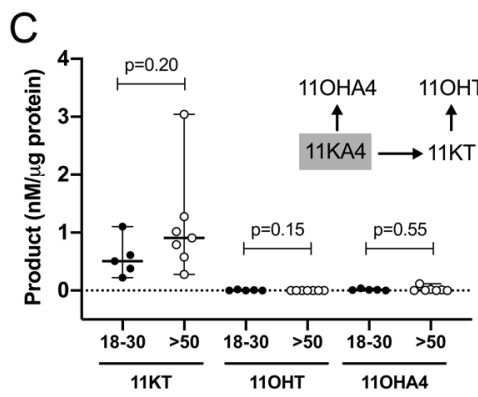

$\mathrm{F}$

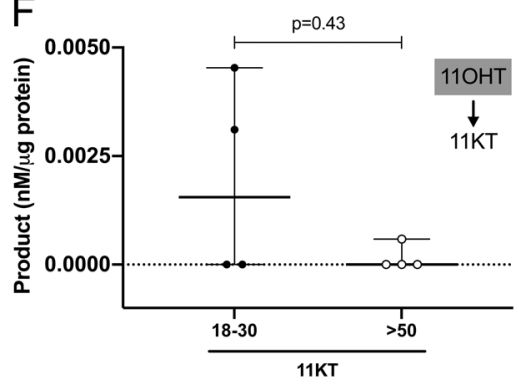

\section{Figure 2}

Ex vivo metabolism of classic ( $A$ and $B$ ) and 11-oxygenated androgens ( $C, D, E$ and $F$ ) by PBMCs isolated from healthy men aged $18-30$ years ( $n=4-5$; age $22-30$ years; BMI 20.2-29.1 kg/m²; solid circles) and men aged $>50$ years $(n=4-7$; age $53-72$ years; BMI $21.2-30.4 \mathrm{~kg} / \mathrm{m}^{2}$; open circles). PBMCs were incubated with $100 \mathrm{nM}$ substrate for $24 \mathrm{~h}$. The respective substrate is shown in a grey box for each graph. Product formation was quantified by LC-MS/MS and normalized to the total protein content of the incubation. Median and range are indicated. Product concentrations below the limit of quantification are shown as 0 . Statistical analysis was performed using Mann-Whitney test.

times more $11 \mathrm{KT}$ than $\mathrm{T}$ from their respective precursors 11KA4 and A4 (Fig. 3A and C). However, while $\mathrm{T}$ was converted back to A4 in large quantities, incubation with $11 \mathrm{KT}$ led to only minor generation of 11KA4 (quantifiable in $8 / 12$ incubations, Fig. 3D), further contributing to the preferential activation of $11 \mathrm{KT}$ by PBMCs.

Expressing the observed steady-state between activation and inactivation as product/substrate ratios made this difference even more obvious, clearly indicating that the generation of active 11-oxygenated androgens is favored in PBMCs (Fig. 4A). Product/substrate-ratios for different substrates of $5 \alpha$-reductase reflect the established substrate preference for SRD5A1 with A4 resulting in the highest activity followed by $\mathrm{T}$ and with only minor activity for 11OHA4 (Fig. 4B).

The generation of 11OHA4 from 11KA4 (quantifiable in 8/12 incubations) and of $11 \mathrm{OHT}$ from $11 \mathrm{KT}$ (quantifiable in 4/12 incubations) indicated HSD11B1 activity in PBMCs, however, at negligible levels compared to the $17 \beta$-hydroxysteroid dehydrogenase and $5 \alpha$-reductase activities observed (Fig. 3C and D). We did not detect any $5 \alpha$-reduced products of $11 \mathrm{KA} 4$ and $11 \mathrm{KT}$ (11-keto- $5 \alpha-$ androstanedione and 11KDHT) ( $n=3$; data not shown).
Incubation with the 11-oxygenated androgen precursors 11OHA4 and 11OHT led to only negligible product formation compared to the other substrates tested. After $24 \mathrm{~h}$ the generation of the $5 \alpha$-reduced product $11 \mathrm{OH}-5 \alpha$-dione from $11 \mathrm{OHA} 4$ could be quantified, as well as the generation of 11KT from 11OHT (Fig. 3E and F).

\section{AKR1C3 expression and activity in PBMCs is primarily located in natural killer cells}

In order to identify the subpopulation(s) within the PBMC compartment responsible for the AKR1C3-catalyzed androgen activation observed in our PBMC incubations, we used publicly available RNAseq-based gene expression data from 15 FACS-sorted PBMC subpopulations including B-cells, different T-cell populations, monocytes and natural killer (NK) cells (dice-database.org (25)). This revealed significantly higher $A K R 1 C 3$ expression in NK cells $(P<0.0001)$ than in any other PBMC subpopulation (Fig. 5A). There was no difference between AKR1C3 expression in NK cells from male and female donors (Fig. 5B). To confirm NK cells as the major site of AKR1C3 activity, we isolated PBMCs from a leukocyte cone of an 
A

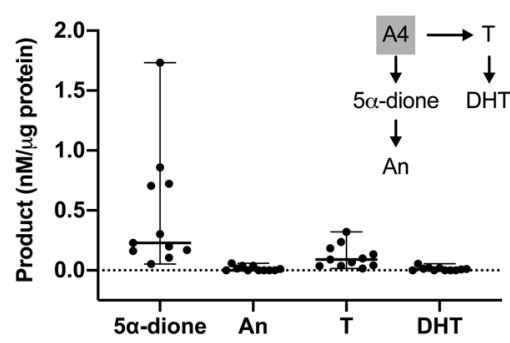

D

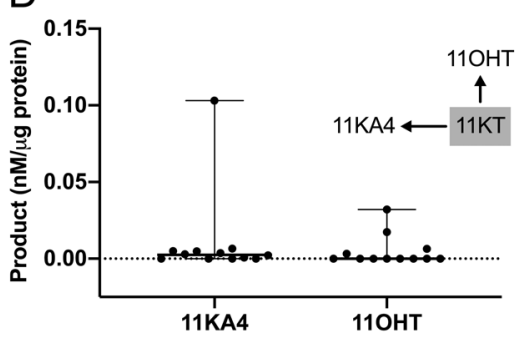

$\mathrm{B}$
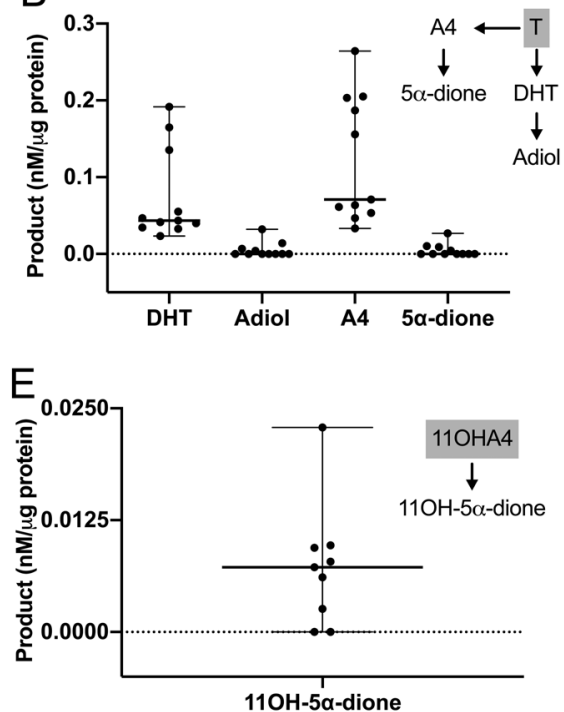
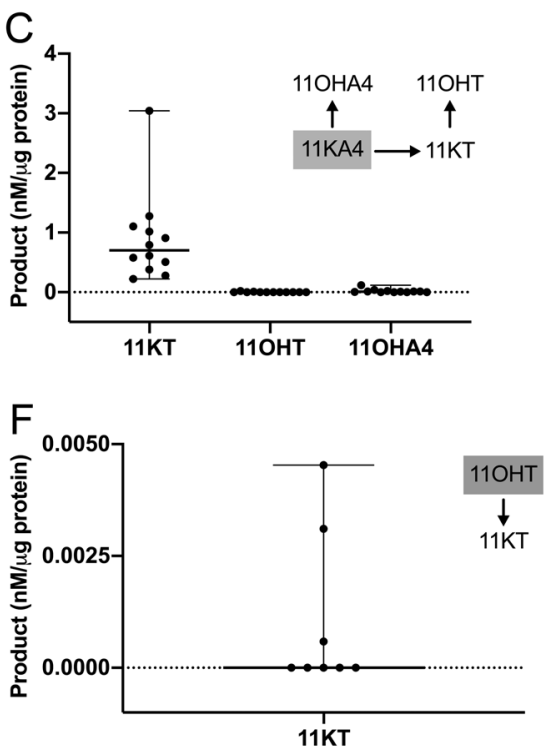

Figure 3

Ex vivo metabolism of classic (A and B) and 11-oxygenated androgens (C, D, E and F) by PBMCs isolated from healthy men ( $n=$ 8-12; age range 22-72 years; BMI range 20.2-30.4 kg/m²). PBMCs were incubated with $100 \mathrm{nM}$ substrate for $24 \mathrm{~h}$. The respective substrate is shown in a grey box for each graph. Product formation was quantified by LC-MS/MS and normalized to the total protein content of the incubation. Median and range are indicated. Product concentrations below the limit of quantification are shown as 0.

anonymous donor and subsequently enriched NK cells in a fraction of the PBMCs. Ex vivo incubations of the matched crude PBMC isolations and the enriched NK cell fraction with the AKR1C3 substrates A4 and 11KA4 confirmed higher AKR1C3 activity in the NK cell-enriched incubation compared to the incubation with crude PBMC isolates (Fig. 5C).

\section{Lag time prior to the separation of cellular components increases 11-ketotestosterone}

We collected blood samples from six additional healthy volunteers (3 male, 3 female; age range 28-50 years; BMI range $20.8-31.1 \mathrm{~kg} / \mathrm{m}^{2}$ ) to assess the effect of an extended incubation of their blood samples unseparated from cellular components on the quantification of serum steroids by LC-MS/MS (Fig. 6). We observed a time-dependent increase in the serum concentrations of the AKR1C3 product $11 \mathrm{KT}$, reaching a median relative increase of $44 \%$ after $24 \mathrm{~h}$, with significant increases in $11 \mathrm{KT}$ already observed after $2 \mathrm{~h}$ of leaving the full blood samples unseparated (Fig. 6I). Additionally, we observed decreases in the concentrations of the AKR1C3 substrates A4 and 11KA4, with median relative decreases of 19 and $34 \%$, respectively, at $24 \mathrm{~h}$. The observed decreases in serum A4 concentrations were statistically significant for the majority of time points assessed $(P<0.05)$, while statistical significance was not reached for the decrease in 11KA4 (Fig. 6C and $\mathrm{H}$ ).

Among the other analytes investigated (DHEA, T, DHT, An, 11OHA4), only 11OHA4 exhibited significant changes over time $(P<0.05)$, with a median relative decrease of $38 \%$ occurring within one hour after blood collection (Fig. 6G).

\section{Discussion}

Androgen signaling is vital for immune cell function by regulating proliferation, differentiation, cytokine production and other pathways $(7,8)$. Intracrine androgen activation from adrenal precursors contributes a large proportion of androgen receptor activation in peripheral target cells and tissues $(17,26)$. While we have previously shown that PBMCs can activate the classic androgen precursor A4 to T via AKR1C3 and further convert $\mathrm{T}$ to the most potent human androgen DHT (6), we show for the first time in this study that PBMCs preferentially activate the 11-oxygenated androgen precursor 11KA4 to its active counterpart $11 \mathrm{KT}$. We show that PBMCs 
A

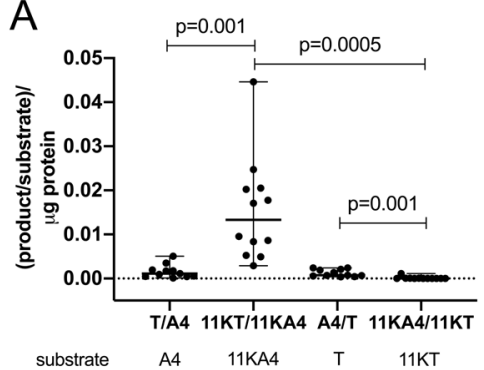

substrate A4 11KA4 $\quad 11 \mathrm{KT}$

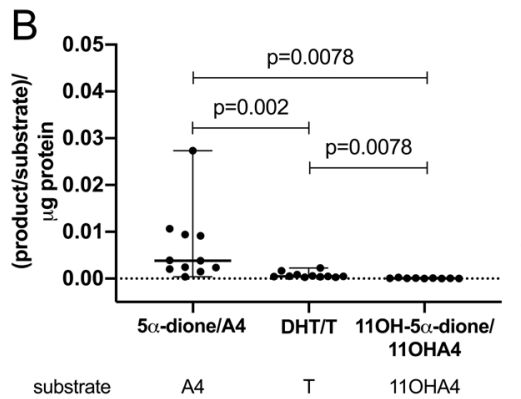

Figure 4

Product/substrate ratios in ex vivo incubations of PBMCs isolated from healthy men ( $n=8-12$; age range $22-72$ years; BMI range $20.2-30.4 \mathrm{~kg} / \mathrm{m}^{2}$ ) after the addition of different steroid substrates. (A) Steady-state ratios of $17 \beta$-hydroxysteroid dehydrogenase products and substrates, reflecting the dominant contribution of the reductive, activating 17 $\beta$-hydroxysteroid dehydrogenase AKR1C3 to 11-oxygenated androgen metabolism. (B) $5 \alpha$-reductase activity by SRD5A1. Product and substrate were quantified by LC-MS/ MS after a 24-h incubation. Ratios were normalized to the total protein content of the incubation. Statistical analysis was performed using the Wilcoxon matched-pairs signed-rank test.

generate approximately 8 -fold more $11 \mathrm{KT}$ than $\mathrm{T}$ from their respective precursors, revealing $11 \mathrm{KT}$ as the predominant active androgen within the human PBMC compartment. Using in vitro systems overexpressing AKR1C3, Barnard et al. (10) previously demonstrated that AKR1C3 has a significantly higher catalytic efficiency for the activation of 11-oxygenated androgens compared to classic androgens, which we have shown here, for the first time, ex vivo in human cells.

In addition, we show that the inactivating conversion of the active androgens $\mathrm{T}$ and $11 \mathrm{KT}$ to their respective precursors A4 and 11KA4 by oxidative $17 \beta$-hydroxysteroid dehydrogenase activity is relevant only for $\mathrm{T}$, while the inactivation of $11 \mathrm{KT}$ to $11 \mathrm{KA} 4$ occurs only in negligible amounts. Taken together, this demonstrates that PBMCs preferentially activate 11-oxygenated androgens. This observation is in agreement with the study by

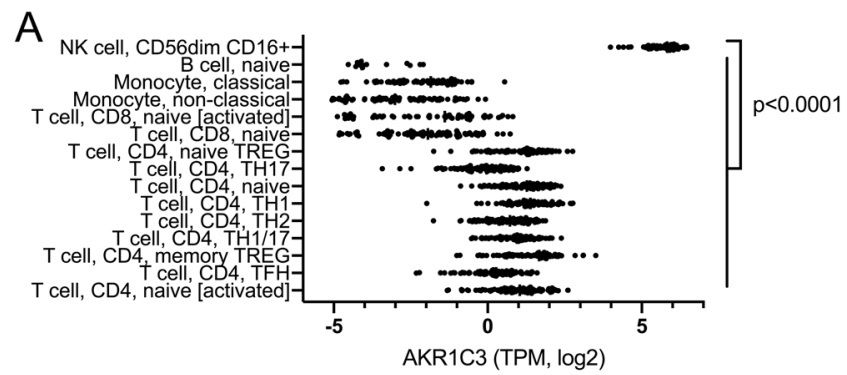

B
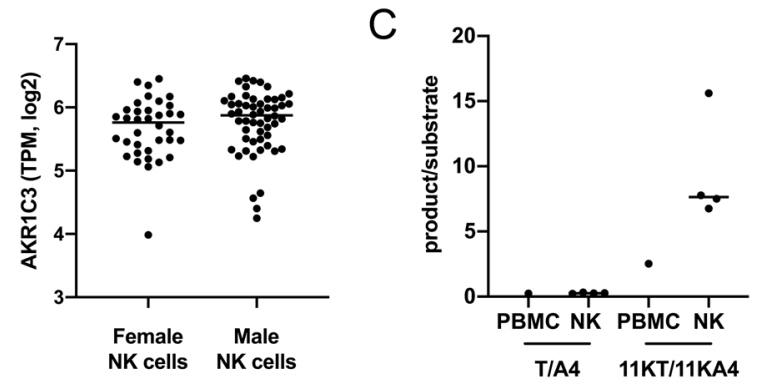

Figure 5

Natural killer cells are the major peripheral blood mononuclear cell population with AKR1C3 expression ( $\mathrm{A}$ and $\mathrm{B}$ ) and activity (C). RNAseq analysis of FACS-sorted PBMC subpopulations (dice-database.org; (25)) revealed natural killer cells (NK cells) as the cell type with the highest AKR1C3 expression (A) with comparable expression in women $(n=37)$ and men $(n=54)$ (B). NK cell-enriched incubations with AKR1C3 substrates ( $n=4$ technical replicates) showed higher AKR1C3 activity (shown as product/substrate ratio) compared to incubations of crude PBMC preparations from the same donor $(n=1)(C)$. NK cells enriched using MACS ${ }^{\circledR}$ negative selection (Miltenyi Biotec) were incubated with $100 \mathrm{nM}$ substrate and product formation after $24 \mathrm{~h}$ was analyzed by LC-MS/MS. Statistical analysis of AKR1C3 expression in the PBMC subpopulation was performed by one-way ANOVA followed by Tukey's multiple comparisons test. AKR1C3 expression in male and female NK cells was compared by Mann-Whitney test. TPM, transcripts per million.

Barnard et al. (10), who showed that, while HSD17B2 catalyzes the inactivation of $\mathrm{T}$ and $11 \mathrm{KT}$ with similar efficiencies, increased ratios of AKR1C3 to HSD17B2 favor the activation of 11-oxygenated androgens due to the catalytic preference of AKR1C3 for 11KA4 over A4.

Our results confirm previous findings that steroid $5 \alpha$-reduction contributes to the activation of classic androgens in PBMCs by generating the most potent human androgen DHT from T (6). The observed substrate preference of $5 \alpha$-reduction for A4 over $\mathrm{T}$ is consistent with the established substrate preference of SRD5A1 (27), which we confirm is the major steroid $5 \alpha$-reductase in 
A

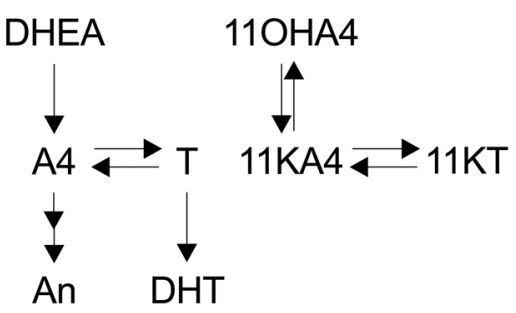

B
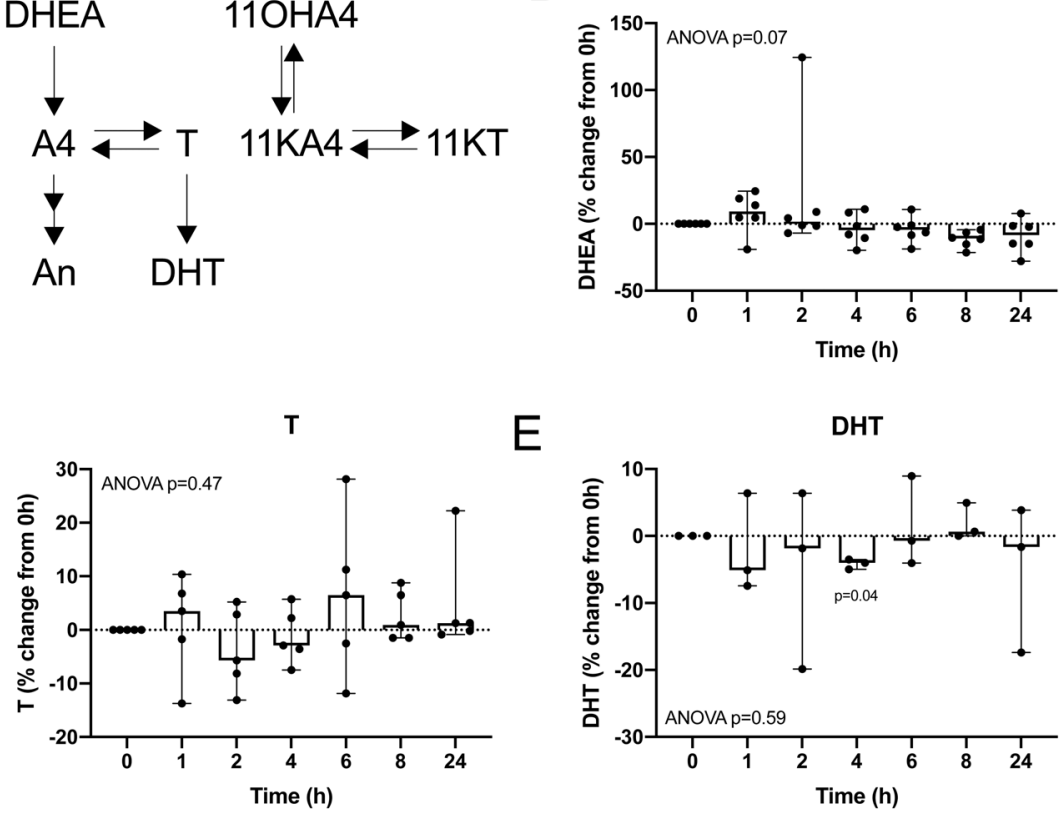

110HA4

G

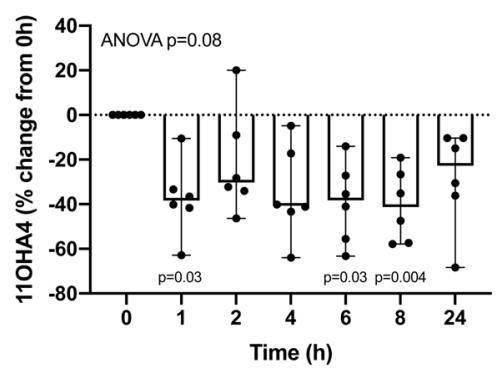

E

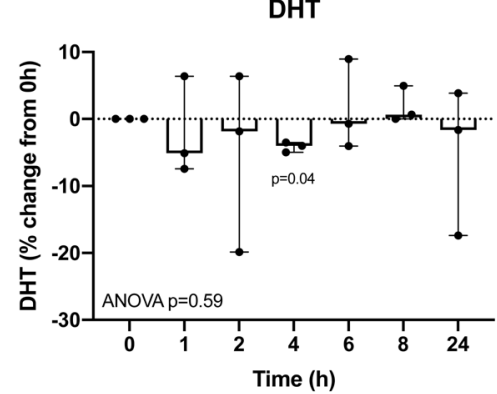

$H$

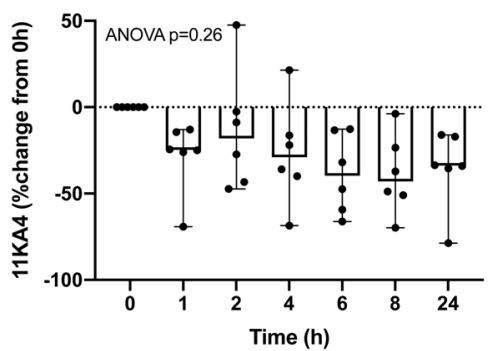

C

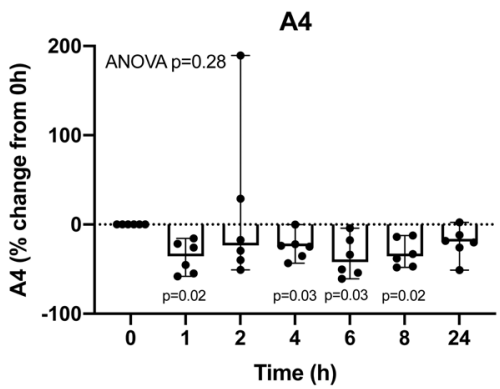

F

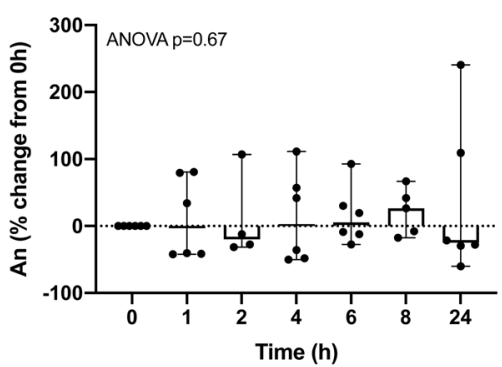

$11 \mathrm{KT}$

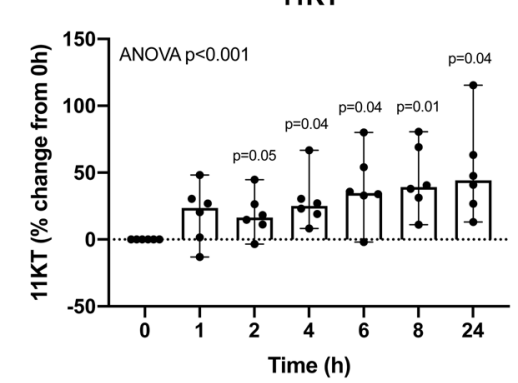

Figure 6

Relative changes in androgen concentrations compared to baseline, measured in serum after incubation of unseparated whole blood samples. Baseline samples were separated by centrifugation within one hour after collection. The remaining samples were incubated as whole blood at room temperature for the times indicated prior to the separation of serum and cellular content (sample numbers: $n=6$ for DHEA (B), $n=6$ for A4 (C), $n=5$ for T (D), $n=3$ for DHT (E), $n=6$ for An (F), $n=6$ for $110 H A 4$ (G), $11 \mathrm{KA} 4$ $(\mathrm{H})$ and $11 \mathrm{KT}(\mathrm{I}))$. Individual data points are shown with range and median. Statistical analysis was performed on the concentrations in nM using ANOVA, followed by Dunnett multiple comparison test to compare each time point against baseline.

PBMCs (6). We did not observe relevant $5 \alpha$-reduction of 11-oxygenated androgens. Using in vitro promoter reporter assays $11 \mathrm{KDHT}$ has been shown to activate the androgen receptor with potency and efficacy comparable to DHT. However, it is not clear if the generation of $11 \mathrm{KDHT}$ by the $5 \alpha$-reduction of $11 \mathrm{KT}$ is relevant under physiological conditions. Recently, we showed that $11 \mathrm{KT}$ metabolism primarily proceeds via the AKR1D1 mediated $5 \beta$-reduction of the steroid A-ring and that SRD5A2, but not SRD5A1, can efficiently catalyze the $5 \alpha$-reduction of $11 \mathrm{KT}$ (28). We now confirm that $11 \mathrm{KT}$ is not $5 \alpha$-reduced by human cells with SRD5A1 levels that efficiently convert T to DHT, confirming that the $5 \alpha$-reduction of $11 \mathrm{KT}$ would require the expression of SRD5A2.

In this study, we did not observe a significant effect of age on androgen activation in PBMCs, with only a trend for increased median AKR1C3 activity, while a previous study by our group described significantly increased AKR1C3 and SRD5A1 activities in men aged over 50 years compared to men aged 18-30 years (6). The limited sample numbers and differences in the assays used for steroid quantification (LC-MS/MS in the current study vs thin 
layer chromatography in the previous one) are likely to be responsible for this discrepancy. However, when assessing the effect of age on androgen activation in peripheral tissues, age-related changes in the supply of androgen precursors from circulation need to be considered, in addition to potential age-dependent changes in the expression of androgen-activating and -inactivating enzymes in the peripheral tissues. While circulating levels of classic androgens significantly decline with age, levels of 11-oxygenated androgens remain constant across adulthood $(29,30)$. Hence, the peripheral activation of 11-oxygenated androgens is favored over the activation of classic androgens not only by the substrate preference of the key androgen-activating enzyme AKR1C3, but also by constant high substrate availability across the life span.

Primary adrenal insufficiency is associated with an increased risk of infection compared to the general population (31). In addition, patients with primary adrenal insufficiency show significantly reduced NK cell cytotoxicity (32). Interestingly, this finding is not affected by DHEA replacement therapy excluding the deficiency of the classic androgen pathway precursor DHEA as a cause of the reduced NK cell cytotoxicity. Our study identifies adrenal 11-oxygenated androgen precursors rather than classic androgen precursors as predominant androgens activated in the PBMC compartment and particularly in NK cells, potentially linking the lack of adrenal 11-oxygenated androgen precursors in primary adrenal insufficiency to the observed decrease in NK cell cytotoxicity.

The preference of PBMCs to generate 11KT from 11KA4 via AKR1C3 activity is further reflected in the significant increases in $11 \mathrm{KT}$ serum concentrations, if cellular components were not removed from the whole blood samples in a timely fashion, confirming previous preliminary observations (23). We found that these changes became significant after two hours at room temperature without separation, suggesting that blood samples for the measurement of 11-oxygenated androgens should be processed within two hours of collection. 11KT is the dominant circulating active androgen in women with polycystic ovary syndrome (15) and congenital adrenal hyperplasia $(14,33)$ and a useful marker for the diagnosis of androgen excess. The ongoing activation of $11 \mathrm{KT}$ in unseparated full blood samples suggests that saliva, a cell-free biofluid, could be a superior matrix for the measurement of $11 \mathrm{KT}(33,34)$.

In conclusion, we show that 11-oxygenated androgen precursors are the preferred substrates for androgen activation in PBMCs, yielding $11 \mathrm{KT}$ as the major active androgen in the PBMC compartment. This is catalyzed by AKR1C3, which is predominantly expressed in NK cells, potentially linking adrenal 11-oxygenated androgen deficiency to the reduced NK cell cytotoxicity in primary adrenal insufficiency. Androgen metabolism by PBMCs can affect the measurement results of $11 \mathrm{KT}$ serum concentrations, if the cellular components of whole blood samples are not removed in a timely fashion.

\section{Declaration of interest}

Wiebke Arlt is on the editorial board of EJE. Wiebke Arlt was not involved in the review or editorial process for this paper, on which she is listed as an author.

\section{Funding}

This work was supported by the Wellcome Trust (Investigator Grant WT209492/Z/17/Z, to W A), the Academy of Medical Sciences UK (Newton Advanced Fellowship NAF004\1002, to K HS) and Diabetes UK (RD Lawrence Fellowship, to I A). WA receives support from the NIHR Birmingham Biomedical Research Centre at the University Hospitals Birmingham NHS Foundation Trust and the University of Birmingham (Grant Reference Number BRC-1215-20009). The views expressed are those of the authors and not necessarily those of the NIHR UK or the Department of Health and Social Care UK.

\section{Acknowledgements}

The authors thank all volunteers for the donation of blood samples. The authors would like to thank Professors Martin Hewison and Karim Raza, University of Birmingham, for their help with obtaining ethical approval for the blood cone collections. The authors are grateful to Emily Powell, University of Birmingham, for her help with the PBMC and NK cell isolation.

\section{References}

1 Penning TM, Burczynski ME, Jez JM, Hung CF, Lin HK, Ma H, Moore M, Palackal N \& Ratnam K. Human 3alpha-hydroxysteroid dehydrogenase isoforms (AKR1C1-AKR1C4) of the aldo-keto reductase superfamily: functional plasticity and tissue distribution reveals roles in the inactivation and formation of male and female sex hormones. Biochemical Journal 2000351 67-77. (https://doi. org/10.1042/0264-6021:3510067)

2 Penning TM, Wangtrakuldee P \& Auchus RJ. Structural and functional biology of aldo-keto reductase steroid-transforming enzymes. Endocrine Reviews 201940 447-475. (https://doi. org/10.1210/er.2018-00089)

3 Schiffer L, Arlt W \& Storbeck KH. Intracrine androgen biosynthesis, metabolism and action revisited. Molecular and Cellular Endocrinology 2018465 4-26. (https://doi.org/10.1016/j.mce.2017.08.016)

4 Zhou Z, Shackleton CH, Pahwa S, White PC \& Speiser PW. Prominent sex steroid metabolism in human lymphocytes. Molecular and Cellular Endocrinology 1998138 61-69. (https://doi.org/10.1016/ s0303-7207(98)00052-5)

5 Milewich L, Kaimal V \& Toews GB. Androstenedione metabolism in human alveolar macrophages. Journal of Clinical Endocrinology and Metabolism 198356 920-924. (https://doi.org/10.1210/jcem-56-5-920) 
6 Hammer F, Drescher DG, Schneider SB, Quinkler M, Stewart PM, Allolio B \& Arlt W. Sex steroid metabolism in human peripheral blood mononuclear cells changes with aging. Journal of Clinical Endocrinology and Metabolism 200590 6283-6289. (https://doi. org/10.1210/jc.2005-0915)

7 Trigunaite A, Dimo J \& Jorgensen TN. Suppressive effects of androgens on the immune system. Cellular Immunology 2015294 87-94. (https://doi.org/10.1016/j.cellimm.2015.02.004)

8 Gilliver SC. Sex steroids as inflammatory regulators. Journal of Steroid Biochemistry and Molecular Biology 2010120 105-115. (https://doi. org/10.1016/j.jsbmb.2009.12.015)

9 Rubinow KB. An intracrine view of sex steroids, immunity, and metabolic regulation. Molecular Metabolism 201815 92-103. (https:// doi.org/10.1016/j.molmet.2018.03.001)

10 Barnard M, Quanson JL, Mostaghel E, Pretorius E, Snoep JL \& Storbeck KH. 11-Oxygenated androgen precursors are the preferred substrates for aldo-keto reductase 1C3 (AKR1C3): implications for castration resistant prostate cancer. Journal of Steroid Biochemistry and Molecular Biology 2018183 192-201. (https://doi.org/10.1016/j. jsbmb.2018.06.013)

11 Pretorius E, Africander DJ, Vlok M, Perkins MS, Quanson J \& Storbeck KH. 11-Ketotestosterone and 11-Ketodihydrotestosterone in castration resistant prostate cancer: potent androgens which can no longer be ignored. PLOS ONE 201611 e0159867. (https://doi. org/10.1371/journal.pone.0159867)

12 Rege J, Turcu AF, Kasa-Vubu JZ, Lerario AM, Auchus GC, Auchus RJ, Smith JM, White PC \& Rainey WE. 11-Ketotestosterone is the dominant circulating bioactive androgen during normal and premature adrenarche. Journal of Clinical Endocrinology and Metabolism 2018103 4589-4598. (https://doi.org/10.1210/jc.2018-00736)

13 Rege J, Nakamura Y, Satoh F, Morimoto R, Kennedy MR, Layman LC, Honma S, Sasano H \& Rainey WE. Liquid chromatography-tandem mass spectrometry analysis of human adrenal vein 19-carbon steroids before and after ACTH stimulation. Journal of Clinical Endocrinology and Metabolism 201398 1182-1188. (https://doi. org/10.1210/jc.2012-2912)

14 Turcu AF, Nanba AT, Chomic R, Upadhyay SK, Giordano TJ Shields JJ, Merke DP, Rainey WE \& Auchus RJ. Adrenal-derived 11-oxygenated 19-carbon steroids are the dominant androgens in classic 21-hydroxylase deficiency. European Journal of Endocrinology 2016174 601-609. (https://doi.org/10.1530/EJE-15-1181)

15 O'Reilly MW, Kempegowda P, Jenkinson C, Taylor AE, Quanson JL, Storbeck KH \& Arlt W. 11-Oxygenated C19 steroids are the predominant androgens in polycystic ovary syndrome. Journal of Clinical Endocrinology and Metabolism 2017102 840-848. (https://doi. org/10.1210/jc.2016-3285)

16 Storbeck KH, Bloem LM, Africander D, Schloms L, Swart P \& Swart AC. 11beta-Hydroxydihydrotestosterone and 11-ketodihydrotestosterone, novel C19 steroids with androgenic activity: a putative role in castration resistant prostate cancer? Molecular and Cellular Endocrinology 2013377 135-146. (https://doi. org/10.1016/j.mce.2013.07.006)

17 Schiffer L, Barnard L, Baranowski ES, Gilligan LC, Taylor AE, Arlt W, Shackleton CHL \& Storbeck KH. Human steroid biosynthesis, metabolism and excretion are differentially reflected by serum and urine steroid metabolomes: a comprehensive review. Journal of Steroid Biochemistry and Molecular Biology 2019194 105439. (https://doi. org/10.1016/j.jsbmb.2019.105439)

18 Turcu AF, Rege J, Auchus RJ \& Rainey WE. 11-Oxygenated androgens in health and disease. Nature Reviews: Endocrinology 202016 284-296. (https://doi.org/10.1038/s41574-020-0336-x)

19 Hammer EJ \& Astley JP. Increase in serum testosterone following contact with blood cells. Annals of Clinical Biochemistry 198522 539-540. (https://doi.org/10.1177/000456328502200513)
20 Jones ME, Folkerd EJ, Doody DA, Iqbal J, Dowsett M, Ashworth A $\&$ Swerdlow AJ. Effect of delays in processing blood samples on measured endogenous plasma sex hormone levels in women. Cancer Epidemiology, Biomarkers and Prevention 200716 1136-1139. (https:// doi.org/10.1158/1055-9965.EPI-07-0028)

21 Zhang DJ, Elswick RK, Miller WG \& Bailey JL. Effect of serumclot contact time on clinical chemistry laboratory results. Clinical Chemistry 199844 1325-1333. (https://doi.org/10.1093/ clinchem/44.6.1325)

22 Hepburn S, Wright MJ, Boyder C, Sahertian RC, Lu B, Zhang R, White CP \& Horvath AR. Sex steroid hormone stability in serum tubes with and without separator gels. Clinical Chemistry and Laboratory Medicine 201654 1451-1459. (https://doi.org/10.1515/ cclm-2015-1133)

23 Hawley JM, Adaway JE, Owen LJ \& Keevil BG. Development of a total serum testosterone, androstenedione, 17-hydroxyprogesterone, $11 \beta$-hydroxyandrostenedione and 11-ketotestosterone LC-MS/MS assay and its application to evaluate pre-analytical sample stability. Clinical Chemistry and Laboratory Medicine 202058 741-752. (https://doi.org/10.1515/cclm-20190959)

24 Quanson JL, Stander MA, Pretorius E, Jenkinson C, Taylor AE \& Storbeck KH. High-throughput analysis of 19 endogenous androgenic steroids by ultra-performance convergence chromatography tandem mass spectrometry. Journal of Chromatography: B, Analytical Technologies in the Biomedical and Life Sciences 20161031 131-138. (https://doi.org/10.1016/j.jchromb.2016.07.024)

25 Schmiedel BJ, Singh D, Madrigal A, Valdovino-Gonzalez AG, White BM, Zapardiel-Gonzalo J, Ha B, Altay G, Greenbaum JA, McVicker G, et al. Impact of genetic polymorphisms on human immune cell gene expression. Cell 2018175 1701.e16-1715.e16. (https://doi.org/10.1016/j.cell.2018.10.022)

26 Labrie F. All sex steroids are made intracellularly in peripheral tissues by the mechanisms of intracrinology after menopause. Journal of Steroid Biochemistry and Molecular Biology 2015145 133-138. (https:// doi.org/10.1016/j.jsbmb.2014.06.001)

27 Thigpen AE, Cala KM \& Russell DW. Characterization of Chinese hamster ovary cell lines expressing human steroid 5 alpha-reductase isozymes. Journal of Biological Chemistry 1993268 17404-17412. (https://doi.org/10.1016/S00219258(19)85349-8)

28 Barnard L, Nikolaou N, Louw C, Schiffer L, Gibson H, Gilligan LC, Gangitano E, Snoep J, Arlt W, Tomlinson JW, et al. The A-ring reduction of 11-ketotestosterone is efficiently catalysed by AKR1D1 and SRD5A2 but not SRD5A1. Journal of Steroid Biochemistry and Molecular Biology 2020202 105724. (https://doi.org/10.1016/j. jsbmb.2020.105724)

29 Davio A, Woolcock H, Nanba AT, Rege J, O'Day P, Ren J, Zhao L, Ebina H, Auchus R, Rainey WE, et al. Sex differences in 11-oxygenated androgen patterns across adulthood. Journal of Clinical Endocrinology and Metabolism 2020105 e2921-9. (https://doi. org/10.1210/clinem/dgaa343)

30 Nanba AT, Rege J, Ren J, Auchus RJ, Rainey WE \& Turcu AF. 11-Oxygenated C19 steroids do not decline with age in women. Journal of Clinical Endocrinology and Metabolism 2019104 2615-2622. (https://doi.org/10.1210/jc.2018-02527)

31 Tresoldi AS, Sumilo D, Perrins M, Toulis KA, Prete A, Reddy N, Wass JAH, Arlt W \& Nirantharakumar K. Increased infection risk in Addison's disease and congenital adrenal hyperplasia. Journal of Clinical Endocrinology and Metabolism 2020105 418-429. (https://doi. org/10.1210/clinem/dgz006)

32 Bancos I, Hazeldine J, Chortis V, Hampson P, Taylor AE, Lord JM \& Arlt W. Primary adrenal insufficiency is associated with impaired natural killer cell function: a potential link to increased mortality. 
European Journal of Endocrinology 2017176 471-480. (https://doi. org/10.1530/EJE-16-0969)

33 Bacila I, Adaway J, Hawley J, Mahdi S, Krone R, Patel L, Alvi S, Randell T, Gevers E, Dattani M, et al. Measurement of salivary adrenal-specific androgens as biomarkers of therapy control in 21-hydroxylase deficiency. Journal of Clinical Endocrinology and
Metabolism 2019104 6417-6429. (https://doi.org/10.1210/jc.201900031)

34 Schiffer L, Adaway JE, Arlt W \& Keevil BG. A liquid chromatographytandem mass spectrometry assay for the profiling of classical and 11-oxygenated androgens in saliva. Annals of Clinical Biochemistry 201956 564-573. (https://doi.org/10.1177/0004563219847498)

Received 24 September 2020

Revised version received 29 November 2020

Accepted 12 January 2021 